\title{
Antireflux mucosectomy band in treatment of refractory gastroesophageal reflux disease: a pilot study for safety, feasibility and symptom control
}

다(1) $(9)$

\author{
Authors

\section{Institutions} \\ 1 Department of Hepatogastroenterology, Université \\ catholique de Louvain, Cliniques Universitaires Saint- \\ Luc, Brussels, Belgium \\ 2 Department of Hepatogastroenterology, Assistance \\ Publique des Hôpitaux de Marseille, Aix-Marseille \\ Université, Hôpital Nord, Marseille, France
}

Laurent Monino ${ }^{1,2}$, Jean-Michel Gonzalez², Véronique Vitton², Marc Barthet ${ }^{2}$ submitted 15.7.2019

accepted after revision 9.10.2019

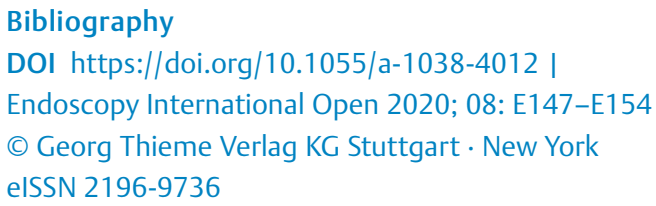

Corresponding author Laurent Monino, MD, Department of Hepatogastroenterology, AP-HM, Aix-Marseille Université, Hôpital Nord, Chemin des Bourrely, 13015 Marseille, France Fax: +33-4-91968737

laurent.monino@uclouvain.be

\section{ABSTRACT}

Background and study aims Antireflux mucosectomy band ligation (ARM-b) is an endoscopic procedure that mimics a fundoplication for managing gastroesophoageal re- flux disease (GERD). The aim of this study was to assess safety and feasibility of ARM-b.

Patients and methods This was a single-center study on consecutive patients operated between June 2017 and January 2019 with refractory GERD, confirmed with pH-metry and without motility disorder at high-resolution manometry. A piecemeal mucosectomy of three quarters of circumference of esophagogastric junction was performed using a band ligation system and a hexagonal snare. The primary objective was to assess procedural safety and feasibility. The secondary objective was to document the clinical improvement at 3 and 6 months based on patient satisfaction, proton pump inhibitor (PPI) intake, symptoms, and quality of life scores.

Results Twenty-onepatients (11 men) with mean age 56.9 \pm 14.4 years were analyzed. The technical success rate was $100 \%$ (mean duration $35 \pm 11 \mathrm{~min}$ ). Four patients (19\%) had mild adverse events: one delayed bleeding at Day 1 managed conservatively, and three dysphagia endoscopically managed. Mean follow-up was $10 \pm 5$ months. Decrease/discontinuation of PPI intake was $76 \%$ at 3 months and $72 \%$ at 6 months. Improvement in mean symptoms and quality of life scores (GERD-Q and GERD-HQL) were statistically significant. One patient required laparoscopic fundoplication after ARM-b failure without complication.

Conclusion ARM-b is safe, feasible, and symptom-effective for treating refractory GERD, and it can be performed in the ambulatory setting. Further prospective studies are required to confirm these promising outcomes.

\section{Introduction}

Gastroesophageal reflux disease (GERD) is one of the most common digestive disease in western countries [1]. For typical GERD without alarm symptoms, proton pump inhibitor (PPI) therapy is a reasonable approach to treat patients combined with lifestyles changes, as stated in American Society of Gastrointestinal Endoscopy (ASGE) recommendations [2,3]. For patients with atypical symptoms, alarm symptoms or who do not respond to PPI therapy, upper endoscopy is recommended for differentiating erosive disease (ERD), non-erosive disease (NERD) and other upper lesions. In patients with NERD, ASGE recommends performing ambulatory $\mathrm{pH}$-impedance monitoring to determine the presence of abnormal esophageal acid or non-acid exposure [3]. Refractory GERD (rGERD), defined as incomplete response after optimization of PPI therapy and abnormal esophageal acid exposure, occurs in $30 \%$ to $40 \%$ of patients [4] and is associated with impaired quality of life [5-8].

In patients with GERD uncontrolled under optimized PPI therapy, ASGE suggests surgical treatment such as laparoscopic 
fundoplication [3] tor reduce the caliber of the EG]. In this situation, laparoscopic Nissen fundoplication is more effective than PPI in mild and long term follow up [9]. However, there is a non-negligible morbidity like dysphagia, inability to blech or vomit and increase bloating and flatulence, called gas bloat syndrome [10]. When surgery is indicated, an esophageal manometry is mandatory for eliminating esophageal mobility disorder.

Few endoscopic treatments have been developed with the aim of reducing the diameter of the esophagogastric junction (EGJ) and mimicking surgical treatment, but these techniques suffer from a lack of efficacy, high cost, and the need for developing new invasive devices [11-13]. Anti-reflux mucosectomy (ARMS) was first reported by Inoue et al [14]. The purpose of this procedure is to achieve endoscopic fundoplication by submucosal fibrosis induced after extensive mucosectomy of the EG]. This technique could be simplified by use of a mucosal band ligation system, so-called antireflux mucosectomy band ligation (ARM-b) [15].

The aim of our pilot study was to assess the feasibility and safety of ARM-b in patients as a primary objective and symptom control as secondary objective with refractory GERD.

\section{Patients and methods}

\section{Design and population}

This was a pilot study with consecutive cases conducted in Marseille between June 2017 and January 2019. Results in patients with refractory GERD after PPI optimization were analyzed.

Esophageal HRM (Manoscan; Sierra Scientific Instruments Inc, Los Angeles, California, United States) recorded pressure changes using the HRM catheter, which contains 36 circumferential pressure sensors. This record was converted with Mano View analysis software (Sierra Scientific Instruments Inc, Los Angeles, California, United States) in a color-coded readout of esophageal pressure. All records were made according to the Chicago classification [16]. Twenty-four-hour ambulatory $\mathrm{pH}$ metry monitoring was performed using an electrode placed $5 \mathrm{~cm}$ above the lower esophageal sphincter. Esophageal acid exposure and patient symptoms were collected and a potential correlation between esophageal exposure and symptoms was investigated $[17,18]$.

After realization of $\mathrm{pH}$-metry and esophageal manometry and confirmation of refractory GERD, patients had clear, fair and objective information about this new endoscopic procedure and its possible adverse events (AEs) or risks. Patients who were eligible for inclusion were included after giving informed consent. Six months prior to the ARM-b procedure, all patients underwent upper endoscopy, $\mathrm{pH}$-metry and HRM.

Inclusion criteria were age over 18 years, clinical history of GERD for more than 2 years uncontrolled by PPI use, positive $\mathrm{pH}$-metry (after PPI disruption) defined as an acid exposure time (AET, \% time with $\mathrm{pH}<4>6 \%$ ) [19-21], high-resolution manometry (HRM) confirming normal esophageal peristalsis defined by more than $50 \%$ of peristaltic waves associated with relaxation of the lower esophageal sphincter during swallowing [16].
Patients were ineligible if they had a hiatal hernia longer than $2 \mathrm{~cm}$ (or Hill score $>3$ or 4 ), severe esophagitis (defined by an esophagitis C or D according to Los Angeles classification), Barrett's esophagus with dysplasia or relief anomaly. Results of HRM, according to the Chicago classification v3.0, were to confirm absence of an esophageal motility disorder or ineffective esophageal motility defined by $>50 \%$ of ineffective swallows, contraction amplitude $<30 \mathrm{mmHg}$ at pressure sensors positioned 3 or $8 \mathrm{~cm}$ above the low esophageal sphincter [16].

The following clinical data were systematically assessed: age, body mass index (BMI), sex, consummation of tobacco and alcohol, surgical history, diet, PPI therapy, and level of satisfaction.

\section{ARM-b procedure}

All patients were informed about the technique and potential AEs. ARM-b procedures [15] were performed under general anesthesia with tracheal intubation. Patients were positioned on their backs. A large operating channel endoscope $(3.8 \mathrm{~mm})$ was used for the procedure (Fuji, Tokyo, Japan or Pentax, Tokyo, Japan), all equipped with virtual chromoendoscopy (iscan for Pentax (Tokyo, Japan) and BLI/CLI for Fuji scope (Tokyo, Japan). The EGJ was endoscopically assessed to confirm absence of con-

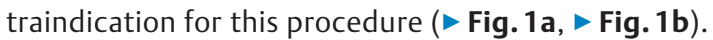

Then a multi-band ligation device (Duette, Cook Medical, Winston Salem, USA) was fitted on the endoscope. The endoscope was positioned at the level of EGJ towards the axis of the lesser curve. Then the procedure was as follows:

1. A 23-G needle was used to inject in the submucosa adrenaline serum (1/1000) for mucosal lifting.

2. The EGJ mucosa was captured with band ligation (1 centimeter in the esophagus and $2 \mathrm{~cm}$ in the stomach).

3. The captured mucosa was cut with a hexagonal snare (Duette, Cook Medical, Winston Salem, North Carolina, United States). The electrosurgical unit setting was Endocut Q, effect 2 (Erbe, Erlangen, Germany).

These three steps were repeated until a piecemeal mucosectomy of three quarters of the circumference of the EG] was achieved.

After retrieving the distal cap, endoscopic assessment of the resected area was conducted to treat potential bleeding or coagulated vessels on the surface. Retroflexure was carried out at the end of the procedure to confirm the complete resection of three-quarters of the circumference of the EG) ( $>$ Fig. 2 and - Fig.3). Technical success was defined by mucosectomy of three-quarters of the EG] circumference in direct vision and retroflexure. All procedures were performed by an endoscopist trained to mucosectomy of upper digestive tract.

During the procedure, a pain-killer protocol (created by anesthesia team) was applied to all patients. It was composed of a minor pain-killer (paracetamol $1 \mathrm{~g}$ ), an antiemetic (metoclopramide) and a PPI (like esomeprazole $40 \mathrm{mg}$ ). 


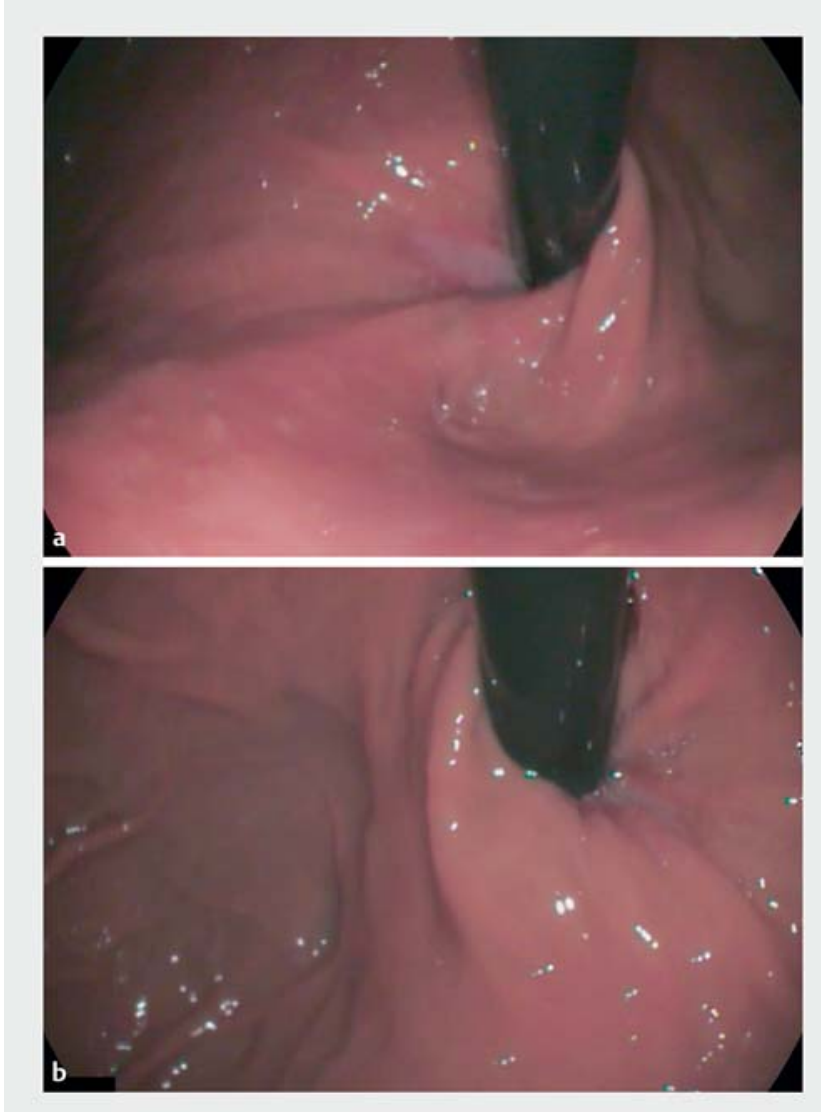

- Fig. 1 a Esophagogastric junction in retroflexion focused on the little curvature of the stomach. b Esophagogastric junction in retroflexion focused on the large curvature of the stomach.

\section{Postoperative course and follow-up}

Patients were monitored for 4 to 6 hours in the recovery room and discharged after the procedure. In case of complication due to the procedure or anesthetic requirement (patient living alone or far away from the hospital), patients were hospitalized.

Patients had a liquid diet on the evening of the procedure and a soft diet for 5 days after the procedure. PPI therapy was continued for 2 months. Each patient was told to modify their diet after the procedure by increasing chewing time and lengthening meal time. Endoscopy was performed at 6 months to assess endoscopic results at EGJ ( $\triangleright$ Fig. $4 \mathbf{a}$ and $\triangleright$ Fig. $4 \mathbf{b}$ ).

During follow-up, patients were called back for medical investigation to be clinically assessed and determine their clinical outcome (PPI intake, symptom score and QOL scores) at 3 and 6 months and then every 6 months thereafter in clinics. Clinical assessment at 3 months was based on:

- Changes in PPI intake, defined as a change in PPI therapy either by discontinuation or a decrease of half-dose;

- Overall satisfaction before/after ARM-b, classified as "good" by a change in satisfaction from dissatisfied to satisfied and "neutral" by a change from neutral to satisfied on a visual analogical scale;

- Improvement in the main GERD symptom with GERD-q [22] before and after ARM-b; and

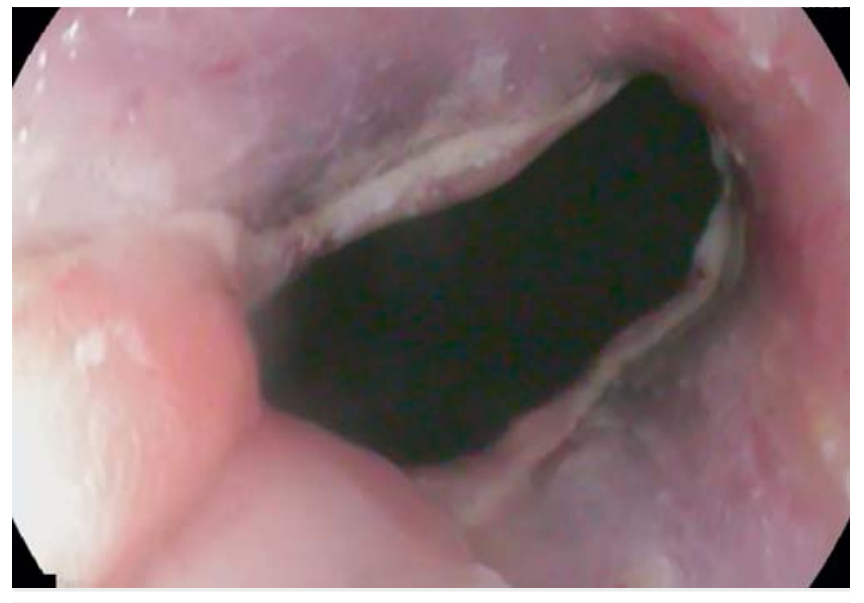

- Fig.2 Direct visualization of esophagogastric junction after ARM-b.

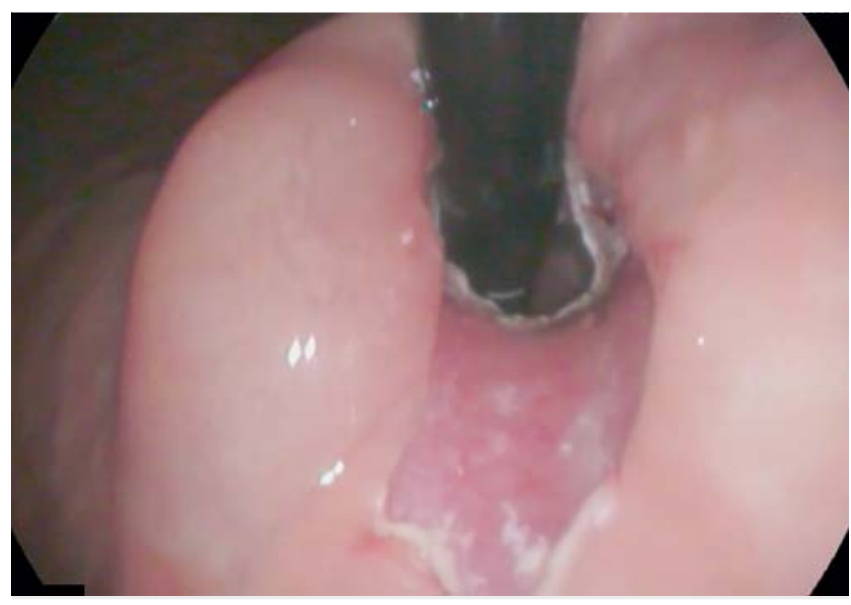

DFig. 3 Esophagogastric junction in retroflexion after ARM-b.

- Evolution of patient quality of life based score GERD-HRQL $[23,24]$ and SF-12 $[25,26]$.

- Clinical assessment at 6 months and every 6 months thereafter was based on:

- Changes in PPI intake, defined as change in PPI therapy either by discontinuation or a decrease of half-dose; and

- Overall satisfaction before/after ARM-b, classified as "good" by a change in satisfaction from dissatisfied to satisfied and "neutral" by a change from neutral to satisfied on a visual analogical scale

\section{Objectives}

The primary objective was to assess the safety and feasibility of the procedure. Judgement criteria were immediate procedural AEs (perforation or bleeding) or delayed (such as esophageal stricture). The secondary objective was to evaluate symptom control with ARM-b. Judgement criteria were:

- main GERD symptom improvement;

- evolution of patient quality of life;

- changes in PPI intake; and

- overall satisfaction before/after ARM-b 


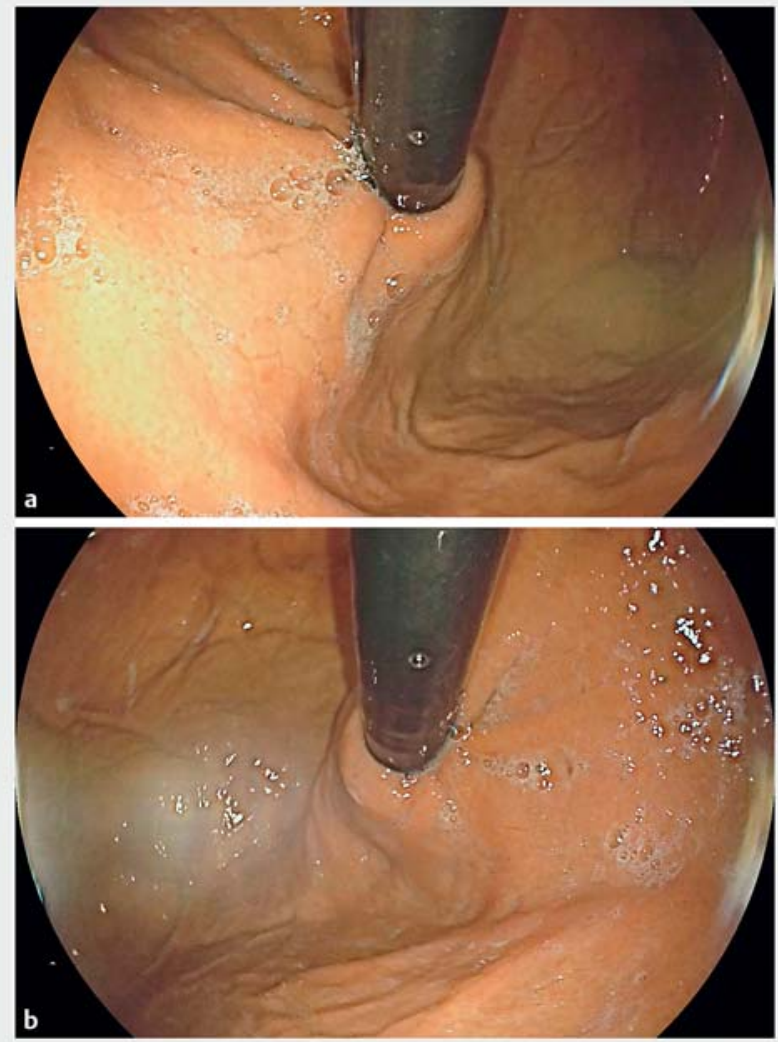

- Fig. 4 a Control of esophagogastric junction in retroflexion after ARM-b focused on the little curvature of the stomach, EGJ narrowing scar. b Control of esophagogastric junction in retroflexion after ARM-b focused on the large curvature of the stomach, EG] narrowing scar.

\section{Regulatory aspects and statistical analysis}

This was a retrospective study $\left(n^{\circ} 2019-176\right)$ in consecutive patients all managed in the same manner with clinical and functional investigations. The data used were anonymized and collected from the Assistance Publique-Hôpitaux de Marseille computer file which is declared to the Commission Nationale Informatique et Liberté (French National Commission for Data Protection). This procedure has been approved by the ethics committee of the AP-HM's digestive disease department.

Qualitative data before/after (Satisfaction score, PPI therapy) were summarized as a percentage and calculated at 3 and 6 months follow-up. For quantitative data before/after (score GERD-q; SF-12; GERD-HRQL), statistical analysis was done with linear regression for calculating coefficient of regression and tested for significance using Student's $t$-test. The regression coefficient $(\alpha)$ must be between 0 and 1 for there to be an improvement after the ARMS-b procedure. If $\alpha$ is close to 0 , the score is modified after performing ARMS-b. $P<0.05$ was considered statistically significant. Data and median of the different scores were represented as a mustache box diagram. Statistical analysis was carried out using TIBO Statistica v13.3 software (licensed granted to the University of Montpellier, research laboratory MRE-TRIS).

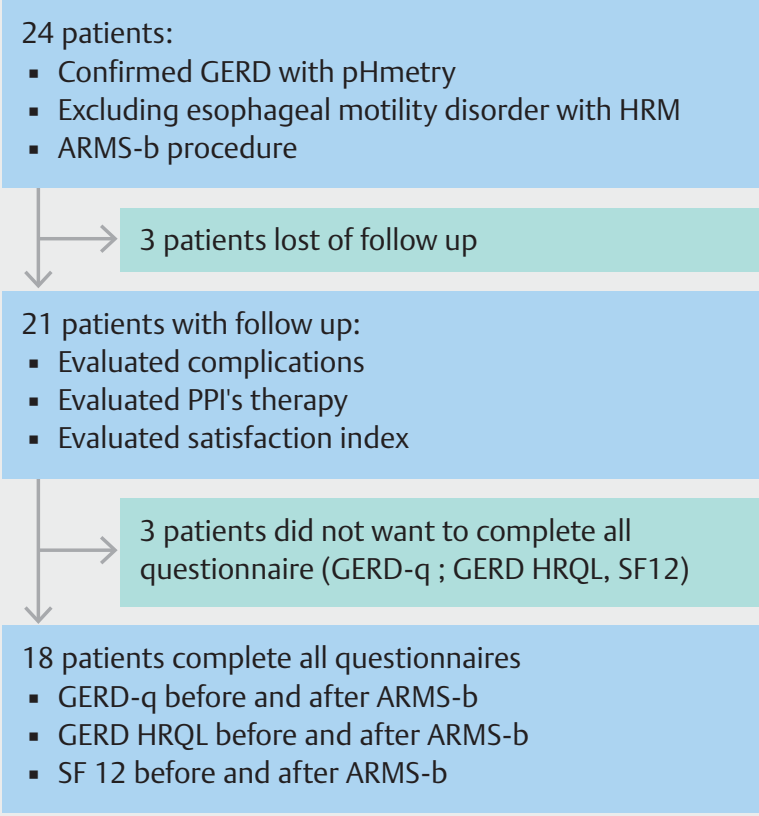

- Fig. 5 Flowchart of study population.

\section{Results}

The ARM-b procedure was performed in 24 patients during the study period between June 2017 and January 2019. Three patients were lost to follow-up ( $\triangleright$ Fig.5). Thus 21 patients -11 men and 10 women - mean age $56.8 \pm 14.4$ years were included in the study. Nineteen patients were treated in the ambulatory setting and three were hospitalized for anesthesia requirements. Patient characteristics are reported in $>$ Table 1 . Before procedure, none of the patients had peptic esophagitis, four patients had Barrett's esophagus (3 COM1 and 1 COM2 according to the Prague classification). All patients had an AET $>6 \%$ by pHmetry.

\section{Procedure safety profile and feasibility}

The technical success rate of ARM-b was $100 \%$ in a mean time of $35 \pm 11$ minutes. No perioperative AEs occurred. One patient had a preventive clipping due to deep resection without perforation or clinical consequences. Endoscopic control at 6 months was achieved in 17 patients (81\%) with a pattern of narrowing of the EGJ scar ( $\mathbf{F i g . 4 a}$ and $\triangleright$ Fig. $\mathbf{4 b}$ ). Of the remaining four patients without narrowing of the EG] scar, one was treated with Nissen surgical fundoplication for persistence of GERD and the remaining three are still receiving PPIs. No reflux esophagitis was reported after the procedure.

Regarding the appearance of EGJ according to Hill's grade, 15 patients had a Hill grade 0 before the procedure. At the endoscopic control at 6 months, 14 those patients had narrowing of the EGJ scar. One patient had a Hill grade 1 before the procedure. For that patient, endoscopic control showed narrowing of the EGJ scar. Five patients had Hill grade 2 before procedure. In them, endoscopic control at 6 month showed no 
change in Hill grade in two patients, a decrease in Hill grade in one patient (1 patient with Hill grade 2 to 1 ) and narrowing of the EGJ scar in the other two.

Postoperative AEs was evaluated in all patients ( $\downarrow$ Table 2 ) and occurred in four patients (19\%). All were Day 1 bleeding, which was non-severe and conservatively managed without blood unit transfusion, endoscopic treatment or hospitalization. Three patients suffered from dysphagia that occurred at 2 to 4 weeks. They were managed by endoscopic hydraulic dilatation (12-mm caliber) requiring one session in one case and two sessions in the two other cases.

\section{Clinical assessment}

Mean time of follow up was $10 \pm 5$ months. Eighteen patients $(85.7 \%)$ were followed for more than 6 months. Patient "good" satisfaction rate was $76 \%$ at 3 months and $72.2 \%$ at 6 months ( $\triangleright$ Table 3 ). Patient rate of change in PPI therapy was $76 \%$ at 3 months and $72.2 \%$ at 6 months, with a complete stop in $57.15 \%$ and $50 \%$ of cases at 3 and 6 months, respectively ( Table 4).

Looking at GERD symptoms and quality of life, 18 patients completed all three questionnaires ( $\triangleright$ Fig.5) before and after undergoing ARMS-b. Three patients refused to complete the questionnaires. Focusing on the main GERD symptoms, median of GERD-Q was 12.5 before the procedure and decreasing to 9 after ( $\triangleright$ Fig.6a).

Regarding quality of life, the median of GERD-HRQL was reduced from 23.5 (with GERD-HRQL heartburn sub-score at 13.5 and regurgitation sub-score at 10.5) to 16.5 (with GERD-HRQL heartburn sub-score at 9 and regurgitation sub-score at 4.5) after the procedure ( $\triangleright$ Fig. $\mathbf{6 b}$ ). As for global quality of life, the median of the mental SF-12 went from 42.58 to 45.83 and the median of physical SF-12 went from 44.24 to 44.72 . In linear regression analysis, the coefficients of regression were statistically significant for GERD-Q ( $\alpha=0.5168$; Student's $t=2.41$; $P=0.0281)$ as well as for GERD-HRQL ( $\alpha=0.4854$; Student's $t=$ $2.22, P=0.041)$. Results of the coefficients of regression $(\alpha)$ for each score are shown in $\nabla$ Table 5 with $P$ values.

\section{Discussion}

In the current study including 21 patients with rGERD treated by ARM-b, the AE rate was $24 \%$ mainly represented by dysphagia with only one early post-procedural complication (bleeding). All AEs were minor as defined by Cotton and al [27] and endoscopically managed with one or two dilations. This result confirmed the safety profile of ARM-b procedure in the ambulatory setting. The dysphagia rate was $14 \%$, which is lower than after surgical fundoplication, as reported in the literature [10]. As a comparison as well, with antireflux endoscopic procedures, serious AEs such as esophageal leaks, hemorrhage, and mediastinal abscess have been reported in the literature with rates between 3.2\% (for TIF) and 13.8\% (for MUSE) [13,28-30]. In our study, this technique was not associated with severe AEs, longer hospital stays or a need for additional endoscopy or surgery. Moreover, the technique failed in only one patient, who required conversion to surgical fundoplication that was without
- Table 1 Characteristics of study population $(n=21)$.

\begin{tabular}{|l|c|}
\hline Age (years) & $56.87( \pm 14.47)$ \\
\hline Male sex & $52.8 \%(11$ men $)$ \\
\hline Average BMI $\left(\mathrm{kg} / \mathrm{m}^{2}\right)$ & $24.35( \pm 4.58)$ \\
\hline Smoke & $23.80 \%(5$ patients $)$ \\
\hline Surgical history & 1 \\
\hline Masson & 1 \\
\hline Gastric bypass & 1 \\
\hline Nissen & 2 \\
\hline Second ARMS-b procedure & $10( \pm 5)$ \\
\hline Average follow-up time (months) & \\
\hline BMI, body mass index; ARMS-b, antireflux mucosectomy band ligation. \\
\hline
\end{tabular}

- Table 2 Adverse events and conversion to surgical fundoplication $(n=21)$.

Global adverse events (\%)

$19 \%$ (4 patients)

Dysphagia (\%)

$14.3 \%$ (3 patients)

Hematemesis without deglobulinization (\%)

Conversion to surgical fundoplication (\%)

$4.8 \%$ ( 1 patients)

$4.8 \%$ (1 patient)

- Table 3 Patient satisfaction.

\begin{tabular}{|l|c|c|}
\hline $\begin{array}{l}\text { Good satisfaction rate at } 3 \\
\text { months }\end{array}$ & At 3 months & At $\mathbf{6}$ months \\
\hline $\begin{array}{l}\text { Dissatisfied or neutral before/ } \\
\text { satisfied after }\end{array}$ & $47.6 \%(10 / 21)$ & $44 \%(8 / 18)$ \\
\hline Dissatisfied before/neutral after & $28.6 \%(6 / 21)$ & $27 \%(5 / 18)$ \\
\hline Neutral before/neutral after & $4.8 \%(1 / 21)$ & $5.5 \%(1 / 18)$ \\
\hline $\begin{array}{l}\text { Dissatisfied before/dissatisfied } \\
\text { after }\end{array}$ & $19 \%(4 / 21)$ & $22 \%(4 / 18)$ \\
\hline
\end{tabular}

Table 4 Change in PPI therapy.

\begin{tabular}{|l|l|l|}
\hline & At 3 months & At $\mathbf{6}$ months \\
\hline Stop or reduction in PPI therapy & $76.2 \%(16 / 21)$ & $72.2 \%(13 / 18)$ \\
\hline Stop PPI therapy & $57.2 \%(12 / 21)$ & $50 \%(9 / 18)$ \\
\hline Reduction in PPI therapy & $19 \%(4 / 21)$ & $22.2 \%(4 / 18)$ \\
\hline Same dose of PPI & $23.8 \%(5 / 21)$ & $27.8 \%(5 / 18)$ \\
\hline
\end{tabular}

PPI, proton pump inhibitor.

technical difficulty. Comparatively, Inoue et al [14] reported a complication rate similar to that for our technique in two of 10 patients requiring endoscopic dilatation after ARMS. 
Mustache box diagram of GERD-HRQL

Median; Box: results between $25-75 \%$; mustache: ranges of results excluding atypia

70

60

50

40

30

20

10

0

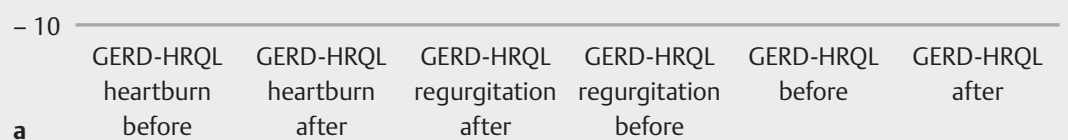

Mustache box diagram of GERD-Q score

Median; Box: results between $25-75 \%$; mustache: ranges

of results excluding atypia

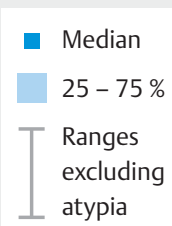

○ Atypical results

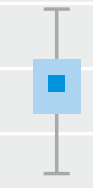

20

18

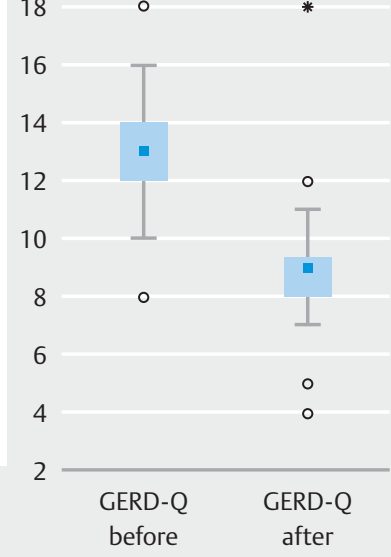

b

- Fig. 6 a Mustache box diagram of median GERD-HRQL. b Mustache box diagram of median GERD-Q.

- Table 5 Statistical analysis of score result with linear regression.

\begin{tabular}{|l|l|l|l|}
\hline Linear regression before/after & Regression coefficient $\mathbf{0 < \boldsymbol { \alpha } \mathbf { 1 }}$ & Student's $\mathbf{~ ( > 2 )}$ & $\mathbf{P}$ value (<0.05) \\
\hline GERD-Q & 0.5168 & 2.4145 & 0.0281 \\
\hline GERD-HRQL heartburn & 0.6595 & 3.5092 & 0.0029 \\
\hline GERD-HRQL regurgitation & 0.4926 & 2.2641 & 0.0378 \\
\hline GERD-HRQL & 0.4854 & 2.2209 & 0.0411 \\
\hline SF-12 physical & 0.9084 & 8.6884 & 0.0000 \\
\hline SF-12 mental & 0.3886 & 1.6871 & 0.1110 \\
\hline GERD-Q, GERD Questionnaire; GERD-HRQL, Gastroesophageal Reflux Disease-Health-Related Quality of Life score. & \\
\hline
\end{tabular}

From a technical point of view, the procedure was designed to achieve "piecemeal" mucosectomy of three-quarters of the EG] circumference and centered on the gastric less curvature, across the $Z$ line involving mainly the gastric mucosa, so as to shorten the EG] diameter. Indeed, as for Barrett's esophagus [31], leaving a healthy mucosal band at the EG] decreases risk of too narrow stricture. Finally, we still recommend submucosal injection for ARM-b, starting on the gastric side of the $Z$ line, to limit risk of full-thickness resection and to suck larger mucosal pieces. Compared to Ithat used by noue et al [14], our ARM-b procedure was more homogeneous than ARMS because Inoue $\mathrm{H}$ et al used two different types of modalities for mucosectomy (endomucosal resection [EMR] and endoscopic submucosal dissection [ESD]) and reported a variable mucosectomy area of EG] (circumferential, two-thirds circumference or half-circumference). In our study, average procedure duration was $35 \pm 11$ minutes compared to 76 minutes for ARMS Cap-EMR from Inoue.

With regard to symptom control, the rate of change in PPI therapy was $76 \%$, with 12 patients stopping the medication completed and four reducing their daily PPI dose at 3 months. Similarly, our results at 6 months are comparable with other endoscopic procedures with a success rate of $72.2 \%$ (TIF: $67 \%$; MUSE: $65 \%$ and STRETTA: $78 \%$ at 6 months) [13]. Unfortunately, because pHmetry data after the procedure are not available, we are unable to demonstrate the complete efficiency of ARM-b.

Analyses of quality of life scores retrospectively calculated before and after ARM-b showed statistically significant changes for GERDq and GERD-HRQL that indicate improvement. As reported by Becher and El Serag $H$ [32], persistence of GERD symptoms despite use of PPIs is associated with a decrease in quality of life. In our study, there was a significant increase in quality of life after the procedure, which suggests better symp- 
tom control. Similarly, there was significant improvement in quality of life related to GERD, as seen with other endoscopic techniques [13]. The lack of statistical significance in mental SF-12 can be explained by the addition of other pathologies disrupting patient quality of life. The regression coefficient for physical SF-12 shows a trend to 1 meaning that ARM-b did not alter the physical quality of life of patients. The "good" rating of satisfaction by only $76 \%$ of patients may reflect difficulty with the question because only one parameter related to quality of life (GERD) was assessed. The statistically significant decrease in GERD-q score after ARM-b clearly illustrates a decrease in typical GERD symptoms (heartburn and regurgitation) which is comparable to Nissen fundoplication [33] and other endoscopic techniques [13]. Use of a score that includes only typical symptoms underscored the effectiveness of our procedure in typical patients. Several studies have shown that in patients with atypical symptoms, surgical fundoplication has fewer effects than in those with typical symptoms [34,35]. These results are encouraging in symptom control and PPI disruption in medium-term follow-up and if confirmed in long-term followup, should demonstrate the efficacy of ARM-b in treatment of GERD.

\section{Conclusion}

In conclusion, ARM-b is safe, reproducible, and feasible in an ambulatory setting, without severe complications. Dysphagia is the main AE associated with this procedure and is easily managed by endoscopic dilatation. PPI disruption was achieved in more than $70 \%$ of patients at 6 months and there was a decrease in healing of GERD symptoms in more than $70 \%$ of patients without recurrence after 6 months. This procedure, which seems simple, could extended to all centers experienced in mucosectomy with band ligation system and would not involve extra costs or a specified learning curve. The encouraging results in this study require longer follow-up and further prospective studies are warranted to confirm the outcomes and determine for which patients ARM-b would be most suitable.

\section{Competing interests}

Professor Barthet and Dr. Gonzalez are consultants for Boston Scientific.

\section{References}

[1] Savarino E, Marabotto E, Bodini G et al. Epidemiology and natural history of gastroesophageal reflux disease. Minerva Gastroenterol Dietol 2017; 63: 175-183

[2] Gyawali CP, Fass R. Management of gastroesophageal reflux disease. Gastroenterology 2018; 154: 302-318

[3] Katz PO, Gerson LB, Vela MF. Guidelines for the diagnosis and management of gastroesophageal reflux disease. Am J Gastroenterol 2013; 108: 308-328

[4] Labenz J, Malfertheiner P. Treatment of uncomplicated reflux disease. World J Gastroenterol 2005; 11: 4291
[5] Yang X-J, Jiang H-M, Hou X-H et al. Anxiety and depression in patients with gastroesophageal reflux disease and their effect on quality of life. World J Gastroenterol 2015; 21: 4302

[6] Lee S-W, Lien H-C, Lee T-Y et al. Heartburn and regurgitation have different impacts on life quality of patients with gastroesophageal reflux disease. World J Gastroenterol 2014; 20: 12277

[7] Mizuki A, Tatemichi M, Sakakibara T et al. A multicenter, randomized, open-label trial: efficacy of once-daily versus twice-daily double-dose rabeprazole on refractory gastroesophageal reflux disease-related symptoms and quality of life. Curr Ther Res Clin Exp 2016; 79: 1

[8] Vakil N, van Zanten SV, Kahrilas P et al. The Montreal definition and classification of gastroesophageal reflux disease: a global evidencebased consensus. Am J Gastroenterol 2006; 101: 1900-1920

[9] Lundell L, Miettinen P, Myrvold HE et al. Comparison of outcomes twelve years after antireflux surgery or omeprazole maintenance therapy for reflux esophagitis. Clin Gastroenterol Hepatol 2009; 7: 1292-1298

[10] Davis CS, Baldea A, Johns JR et al. The evolution and long-term results of laparoscopic antireflux surgery for the treatment of gastroesophageal reflux disease. JSLS J Soc Laparoendosc Surg 2010; 14: 332-341

[11] Ganz RA. A review of new surgical and endoscopic therapies for gastroesophageal reflux disease. Gastroenterol Hepatol (NY) 2016; 12 : 424-31

[12] Pearl J, Pauli E, Dunkin B et al. SAGES endoluminal treatments for GERD. Surg Endosc 2017; 31: 3783-3790

[13] Thosani N, Goodman A, Manfredi M et al. Endoscopic anti-reflux devices (with videos). Gastrointest Endosc 2017; 86: 931-948

[14] Inoue $\mathrm{H}$, Ito $\mathrm{H}$, Ikeda $\mathrm{H}$ et al. Anti-reflux mucosectomy for gastroesophageal reflux disease in the absence of hiatus hernia: a pilot study. Ann Gastroenterol 2014; 27: 346-351

[15] Monino L, Gonzalez J-M, Vitton V et al. Anti-reflux mucosectomy with band ligation in the treatment of refractory gastroesophageal reflux disease. Endoscopy 2019; 51: E215-E216

[16] Kahrilas P], Bredenoord A], Fox M et al. The Chicago Classification of esophageal motility disorders, v3.0. Neurogastroenterol Motil 2015; 27: $160-174$

[17] Kahrilas PJ, Sifrim D. High-resolution manometry and impedance-pH/ manometry: valuable tools in clinical and investigational esophagology. Gastroenterology 2008; 135: 756-769

[18] Savarino E, Tutuian R, Zentilin P et al. Characteristics of reflux episodes and symptom association in patients with erosive esophagitis and nonerosive reflux disease: study using combined impedance-pH off therapy. Am J Gastroenterol 2010; 105: 1053-1061

[19] Picon L, Bruley Des Varannes S. Groupe de Travail sur les Recommandations pour lapratique de la pHmétrie esophagienne. Guidelines for esophageal pH-monitoring in adults. Gastroenterol Clin Biol 2000; 24: 931-943

[20] Roman S, Gyawali CP, Savarino E et al. Ambulatory reflux monitoring for diagnosis of gastro-esophageal reflux disease: Update of the Porto consensus and recommendations from an international consensus group. Neurogastroenterol Motil 2017; 29: e13067

[21] Gyawali CP, Kahrilas PJ, Savarino E et al. Modern diagnosis of GERD: the Lyon Consensus. Gut 2018; 67: 1351-1362

[22] Jones R, Junghard O, Dent J et al. Development of the GerdQ, a tool for the diagnosis and management of gastro-oesophageal reflux disease in primary care. Aliment Pharmacol Ther 2009; 30: 1030-1038

[23] Velanovich V. The development of the GERD-HRQL symptom severity instrument. Dis Esophagus 2007; 20: 130-134

[24] Chassany O, Holtmann G, Malagelada J et al. Systematic review: health-related quality of life (HRQOL) questionnaires in gastro-oesophageal reflux disease. Aliment Pharmacol Ther 2008; 27: 10531070 
[25] Gandek B, Ware JE, Aaronson NK et al. Cross-validation of item selection and scoring for the SF-12 Health Survey in nine countries: results from the IQOLA Project. International Quality of Life Assessment. J Clin Epidemiol 1998; 51: 1171-1178

[26] Brazier JE, Harper R, Jones NM et al. Validating the SF-36 health survey questionnaire: new outcome measure for primary care. BMJ 1992; 305: $160-164$

[27] Cotton PB, Eisen GM, Aabakken L et al. A lexicon for endoscopic adverse events: report of an ASGE workshop. Gastrointest Endosc 2010; 71: 446-454

[28] Wendling MR, Melvin WS, Perry KA. Impact of transoral incisionless fundoplication (TIF) on subjective and objective GERD indices: a systematic review of the published literature. Surg Endosc 2013; 27: 3754-3761

[29] Lipka S, Kumar A, Richter JE. No evidence for efficacy of radiofrequency ablation for treatment of gastroesophageal reflux disease: a systematic review and meta-analysis. Clin Gastroenterol Hepatol 2015; 13: 1058-1067.e1

[30] Zacherl J, Roy-Shapira A, Bonavina L et al. Endoscopic anterior fundoplication with the Medigus Ultrasonic Surgical Endostapler (MUSE ${ }^{\mathrm{TM}}$ ) for gastroesophageal reflux disease: 6 -month results from a multicenter prospective trial. Surg Endosc 2015; 29: 220-229

[31] Pouw RE, Beyna T, Belghazi K et al. A prospective multicenter study using a new multiband mucosectomy device for endoscopic resection of early neoplasia in Barrett's esophagus. Gastrointest Endosc 2018; 88: 647-654

[32] Becher A, El-Serag H. Systematic review: the association between symptomatic response to proton pump inhibitors and health-related quality of life in patients with gastro-oesophageal reflux disease. Aliment Pharmacol Ther 2011; 34: 618-627

[33] Wileman SM, McCann S, Grant AM et al. Medical versus surgical management for gastro-oesophageal reflux disease (GORD) in adults. In: Wileman SM. Cochrane Database of Systematic Reviews. Chichester, UK: John Wiley \& Sons, Ltd;CD003243

[34] Krill JT, Naik RD, Higginbotham T et al. Association between response to acid-suppression therapy and efficacy of antireflux surgery in patients with extraesophageal reflux. Clin Gastroenterol Hepatol 2017; 15: 675-681

[35] Oelschlager BK, Quiroga E, Parra JD et al. Long-term outcomes after laparoscopic antireflux surgery. Am J Gastroenterol 2008; 103: 280287; quiz 288 